\title{
Ulnar nerve abscess in a multibacillary patient during post-multidrug therapy surveillance
}

\author{
SELVASEKAR ABRAHAM, P. VIJAYAKUMARAN \& \\ K. JESUDASAN \\ Schieffelin Leprosy Research \& Training Centre, Karigiri, Vellore Dt., \\ Tamil Nadu, South India-632 106
}

Accepted for publication 7 August 1997

\begin{abstract}
Summary An old borderline-lepromatous leprosy patient, treated initially with DDS monotherapy followed by MDT, 11 years later during surveillance presented with 3-month-old asymptomatic cystic swelling, arising from the right ulnar nerve without exhibiting any evidence to document relapse of the disease. It responded promptly to corticosteroid therapy.

This unusual clinical presentations of ulnar nerve abscess has not been reported elsewhere.
\end{abstract}

\section{Introduction}

Abscesses in the peripheral nerves are not infrequent in patients with leprosy invariably, they occur in the active phase of the disease. We report here an ulnar abscess which developed under rather unusual circumstances.

\section{Case report}

In January 1964, a 26-year-old woman presented with clawing of the fingers of both hands. There were symmetrical, hypopigmented patches over both forearms, back, trunk and buttocks, with fine infiltration over the chin and ears. The ulnar and lateral popliteal nerves were enlarged. Her hands showed flexion and contractures, more pronounced on the right. She also had right foot drop with ulcers on the hands and foot.

She was diagnosed as having indeterminate leprosy. Treatment with thiambutosine was started and the ulcers grafted.

In August 1966 she began treatment with DDS $50 \mathrm{mg} /$ day. Despite intensive re-education on the care of her hands and feet, she developed ulcers of her right hand on several occasions.

In December 1968, she was reclassified as borderline lepromatous leprosy.

Two months later, the patches on her face and body were exacerbated. Her ears were reddish and nodular. Satellite lesions were seen over the patches on her back. There was no neuritis. 
The diagnosis was: relapse and exacerbated BL. Her BI was $1 \cdot 87+$ at that time.

By May 1982, she had developed ulcers on 10 separate occasions and was left with the following deformities: complete resorption of four fingers of the right hand to the level of the metacarpophalangeal joints and of the thumb to the distal interphalangeal joint. All fingers were resorbed to the level of the distal interphalangeal joint on her left hand. She had complete loss of sensation in both feet. The third and fourth toes of the right foot were resorbed.

In June 1982, she was treated with WHO MB-MDT for 24 months. Her skin smears remained negative throughout.

On release from treatment her deformities had increased the right and little fingers of the left hand had resorbed to the proximal interphalangeal joints.

The third, fourth and fifth toes of her right foot were completely lost and the second toe resorbed to the distal interphalangeal joint.

She was assessed annually and had progressive deformities.

In September 1995, she noticed a small swelling above her right elbow. It was painless and she sought advice in December 1995.

Examination showed a $2-\mathrm{cm}$ by $2-\mathrm{cm}$ cystic mass about $10 \mathrm{~cm}$ above her medial epicondyle. The mass moved slightly, perpendicular to the course of the ulnar nerve. There was no inflammation. She had no constitutional signs or symptoms, nor was there any deterioration of sensory function. With her severe deformities, motor assessment was not possible.

Skin smears for acid-fast bacilli from routine sites and over the swelling were negative.

A tentative diagnosis of nerve abscess was made. The patient was started on $40 \mathrm{mg} / \mathrm{day}$ of methyl prednisolone. One week later, the swelling was half its original size, and by the thirtyfourth day of treatment it had almost disappeared, leaving a slight elevation on the ulnar nerve trunk. The steroids were tapered after 2 months.

The patient has shown no sign of relapse up to now.

\section{Discussion}

A nerve biopsy would certainly have clinched the diagnosis in the patient reported here, but was considered unwarranted as the response to treatment was excellent.

Nerve abscesses occur when the disease is active, but this patient had no physical signs to suggest a relapse.

The persistence of a granulomata long after 'cure' has been demonstrated in patients more than three years after cessation of treatment in Hansen's disease. ${ }^{1}$ However it is difficult to assume that this had occurred in our patient 11 years after the completion of treatment. She developed a solitary nerve swelling without regional or constitutional signs and symptoms after 25 years of smear negativity and 11 years after completion of multidrug therapy.

\section{Acknowledgment}

The authors thank Mr Y. Nallathambi, Physiotherapist, Mr S. Vincent, Laboratory Technician, Mr C. Lewis Kumar and Ms J. Helen Jothi for the secretarial assistance and are grateful to the patient for her immense co-operation. 


\section{Reference}

1 Desikan P, Desikan K V. Persistence of lepromatous granuloma in clinically cured cases of leprosy. Int J Lepr 1995; 63: 417-421. 